\title{
Acoustic resolution photoacoustic Doppler velocity measurements in fluids using time-domain cross-correlation
}

\author{
J. Brunker and P. Beard \\ Department of Medical Physics and Bioengineering, University College London, Gower Street, \\ London, WC1E 6BT, United Kingdom \\ http://www.medphys.ucl.ac.uk/research/mle/index.htm
}

\begin{abstract}
Blood flow measurements have been demonstrated using the acoustic resolution mode of photoacoustic sensing. This is unlike previous flowmetry methods using the optical resolution mode, which limits the maximum penetration depth to approximately $1 \mathrm{~mm}$. Here we describe a pulsed time correlation photoacoustic Doppler technique that is inherently flexible, lending itself to both resolution modes. Doppler time shifts are quantified via cross-correlation of pairs of photoacoustic waveforms generated in moving absorbers using pairs of laser light pulses, and the photoacoustic waves detected using an ultrasound transducer. The acoustic resolution mode is employed by using the transducer focal width, rather than the large illuminated volume, to define the lateral spatial resolution. The use of short laser pulses allows depth-resolved measurements to be obtained with high spatial resolution, offering the prospect of mapping flow within microcirculation. Whilst our previous work has been limited to a non-fluid phantom, we now demonstrate measurements in more realistic blood-mimicking phantoms incorporating fluid suspensions of microspheres flowing along an optically transparent tube. Velocities up to $110 \mathrm{~mm} / \mathrm{s}$ were measured with accuracies approaching $1 \%$ of the known velocities, and resolutions of a few $\mathrm{mm} / \mathrm{s}$. The velocity range and resolution are scalable with excitation pulse separation, but the maximum measurable velocity was considerably smaller than the value expected from the detector focal beam width. Measurements were also made for blood flowing at velocities up to $13.5 \mathrm{~mm} / \mathrm{s}$. This was for a sample reduced to $5 \%$ of the normal haematocrit; increasing the red blood cell concentration limited the maximum measurable velocity so that no results were obtained for concentrations greater than $20 \%$ of a physiologically realistic haematocrit. There are several possible causes for this limitation; these include the detector bandwidth and irregularities in the flow pattern. Better results are obtained using a detector with a higher centre frequency and larger bandwidth and tubes with a narrower diameter.
\end{abstract}

Keywords: Doppler, photoacoustic, pulsed, acoustic resolution, cross-correlation, flow, velocity, blood

\section{INTRODUCTION}

Photoacoustic imaging relies upon the use of laser generated ultrasound to produce optical absorption based images of soft tissues [1]. Endogenous image contrast is dominated by haemoglobin on account of its strong optical absorption at visible and near infrared wavelengths. As a consequence, photoacoustic imaging is well suited to providing images of vascular anatomy $[2,3,4]$. In addition, by varying the excitation laser wavelength and exploiting the known spectral differences between oxy- and deoxy-haemoglobin, measurements of absolute blood oxygen saturation can be made [5]. This ability to characterise the structure and oxygenation status of the vasculature makes the technique well suited to the study of tumours and other pathologies characterised by abnormalities in perfusion and oxygen supply. A further potential functional capability is the measurement of blood velocity using Doppler flowmetry techniques. This would be useful in its own right, for example to study flow in tumour vessels where the tortuous nature of the microvasculature can lead to chaotic and variable blood flow which can inhibit therapeutic response. However, if both blood flow and oxygen saturation can be measured simultaneously there is also the prospect of being able to estimate oxygen delivery and thus provide a measure of oxygen consumption - an important physiological parameter that is almost impossible to measure non-invasively using other methods without employing contrast agents.

Photoacoustic flow measurements can be made in a manner analogous to conventional pulse echo Doppler ultrasound that is to say by recovering the Doppler frequency, phase or time shift encoded on to photoacoustic waves emitted by moving red blood cells. Unlike Doppler ultrasound however, the detected acoustic signal is emitted by the blood cells as

Photons Plus Ultrasound: Imaging and Sensing 2013, edited by Alexander A. Oraevsky, Lihong V. Wang, Proc. of SPIE Vol. 8581, 85811U · (C) 2013 SPIE · CCC code: 1605-7422/13/\$18 · doi: 10.1117/12.2004742 
opposed to being weakly reflected from them. This offers significant SNR advantages especially when measuring flow in microvessels as these exhibit low echogenicity. Moreover, Doppler ultrasound measurements of the relatively low flow velocity $(<50 \mathrm{~mm} / \mathrm{s})$ in microvessels can be corrupted by the much larger backscattered signal from the surrounding tissue. In photoacoustic Doppler flowmetry this is likely to be less problematic due to the strong optical absorption of blood compared to that of the neighbouring tissue.

The basic principles of photoacoustic Doppler flowmetry were first outlined over a decade ago [6]. Subsequently, photoacoustic measurements of flow have been obtained by recovering Doppler frequency shifts using CW excitation [7]. However, the use of pulsed excitation [8] is preferable since, unlike CW methods, it readily provides depth-resolved measurements. Pulsed approaches have also been successfully demonstrated in the time domain $[9,10]$, but only in the "optical resolution" mode of photoacoustic sensing, which is limited to a maximum penetration depth of approximately 1 $\mathrm{mm}$. The method has yet to be demonstrated using the acoustic resolution mode that provides much greater penetration depths.

In this paper, we present velocity measurements made in fluid phantoms using a different Doppler flowmetry approach, which is described in detail in reference [11]. The method is based upon time correlation signal processing and involves measuring the change in the time-of-arrival of successive photoacoustic waves emitted by a moving cluster of red blood cells under pulsed excitation using the cross-correlation function. Velocity range and resolution are scalable with excitation pulse separation allowing it be optimised for a wide range of physiologically realistic flow velocities, and it lends itself to both the acoustic and optical resolution modes of photoacoustic sensing. Our previous work has mainly been limited to a non-fluid phantom $[11,12,13]$, but here we assess factors affecting correlation in more realistic fluid phantoms. Section 2 outlines the underlying principles of the technique and section 3 the experimental methods used to evaluate it in blood-simulating phantoms. Section 4 describes the signal processing and methods for estimating the velocity. The experimentally determined velocity measurements, resolution and dynamic range are presented and discussed in section 5 .

\section{PRINCIPLES OF PULSED PHOTOACOUSTIC DOPPLER FLOWMETRY}

Figure 1 shows a schematic that illustrates the concept. A sample of tissue is irradiated with a wide field pulsed laser beam. Photoacoustic waves are emitted predominantly by the red blood cells (RBCs) within a subsurface blood vessel and detected by a directional ultrasound detector located on the tissue surface. Since an extended region of the tissue is illuminated, the focal beam of the detector, rather than the illuminated volume, defines the region over which the signals are collected - the so-called acoustic resolution mode of photoacoustics. This is in contrast to optical resolution mode of photoacoustic microscopy which is limited to a maximum penetration depth of approximately $1 \mathrm{~mm}$.

Measurement of the velocity of blood within a vessel as depicted in Figure 1 requires irradiation with at least two laser pulses separated by a time $T$. During time $T$ the RBCs move a distance $d$ resulting in a time shift $t_{s}$ between the photoacoustic signals $p_{l}(t)$ and $p_{2}(t)$. This measured time shift is related to the speed of sound $c$ in the medium and the angle $\theta$ between the direction of particle motion and the axis of the transducer receive beam:

$$
t_{s}=\frac{d}{c}=\frac{l \cos \theta}{c}
$$

The velocity of particles $V$ is then given by

$$
V=\frac{c t_{s}}{T \cos \theta}
$$

This is analogous to the Doppler equation derived for pulse-echo ultrasound.

The cross-correlation function can be used to determine the time shift $t_{s}$ between the two photoacoustic waveforms $p_{l}(t)$ and $p_{2}(t)$. For discrete $N$-length vectors, an estimate of the unbiased cross-correlation function is given by:

$$
\hat{R}_{\mathbf{p}_{\mathbf{1}} \mathbf{p}_{\mathbf{2}}}(m)= \begin{cases}\frac{1}{N-|m|} \sum_{n=0}^{N-m-1} \mathbf{p}_{\mathbf{1}(n+m)} \mathbf{p}_{\mathbf{2} n}^{*} & m \geq 0 \\ \hat{R}_{\mathbf{p}_{\mathbf{2}} \mathbf{p}_{\mathbf{1}}}^{*}(-m) & m<0\end{cases}
$$

The discretely sampled signals $\mathbf{p}_{\mathbf{k} n}$ are related to their continuous counterparts by $\left.\mathbf{p}_{\mathbf{k} n} \equiv p_{k}(t)\right|_{t=n \Delta t}$. 


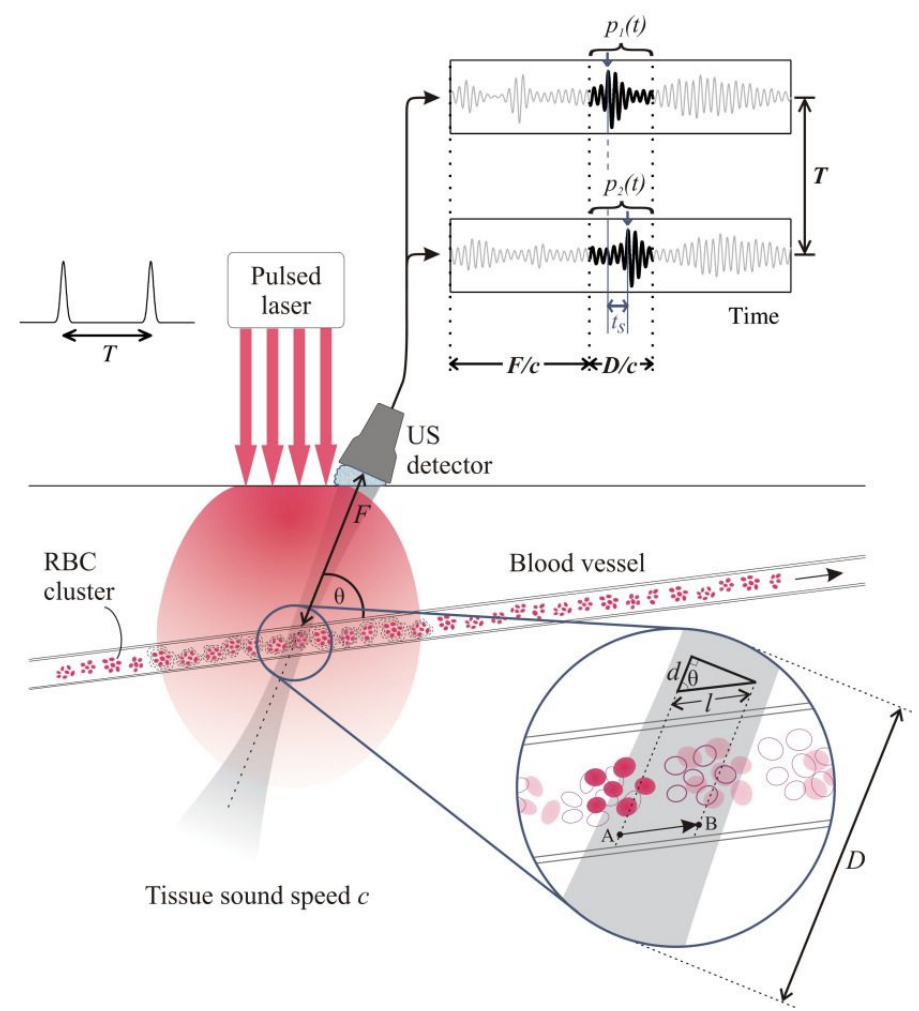

Figure 1. Schematic showing the detection of time-shifted photoacoustic signals. The two signals are generated from clusters of moving red blood cells (RBCs) when illuminated by a pair of laser pulses separated by a time $T$. The inset shows the distribution of RBCs (represented by solid ellipses) when the first laser pulse is fired, and the new positions (unfilled ellipses) coincident with the firing of the second laser pulse a time $T$ later. Between the two pulses the cells have moved from $A$ to $B$, a distance $l$ along the blood vessel. The waveforms show segments of the two photoacoustic signals $p_{I}(t)$ and $p_{2}(t)$ that correspond to the location of the blood vessel and illustrate the time shift $t_{s}$ between the two due to the motion of the RBCs.

\section{EXPERIMENTAL METHOD}

Figure 2 shows the experimental setup used to demonstrate the technique. Fluid phantoms comprising suspensions of phenolic resin microspheres (BRACE $\mathrm{GmbH}$ ) were used to simulate the motion of red blood cells. The solution for suspending the microspheres was made by dissolving an appropriate mass of solid sodium polytungstate (71913, SigmaAldrich) into distilled water so that the mass density of the solution matched that of the microspheres. Three different sizes of microspheres were investigated, with mean particle diameters of $22 \mu \mathrm{m}, 17 \mu \mathrm{m}$ and $6 \mu \mathrm{m}$. Particle aggregation was reduced by adding a 1\% volume of TWEEN@ 20 (P1379, Sigma-Aldrich). Fluid flow was generated using a syringe pump (B. Braun Space ${ }^{\circledR}$ ) with a $60 \mathrm{ml}$ syringe (BD Plastipak ${ }^{\mathrm{TM}}$ ) and a polymer tube (Paradigm Optics). Results are presented for the microspheres flowing in a tube made from THV polymer and with an inner diameter (I.D.) of $800 \mu \mathrm{m}$. Subsequent experiments used human blood diluted to various red cell concentrations (haematocrits) using phosphate buffered saline. Unless otherwise stated, the blood was flowing in a PMMA polymer tube with I.D. $600 \mu \mathrm{m}$. Other tube diameters were also investigated, including one made from PMMA polymer with I.D. $1400 \mu \mathrm{m}$.

Results acquired using a solid phantom are also shown for comparison purposes. This is the "wheel phantom" described in our previous work [11-13] and consists of a random pattern of light-absorbing micron-scale dots rotating near the edge of a Perspex disc. The rotary motion of the dots can be approximated to the linear flow of red blood cell clusters. 


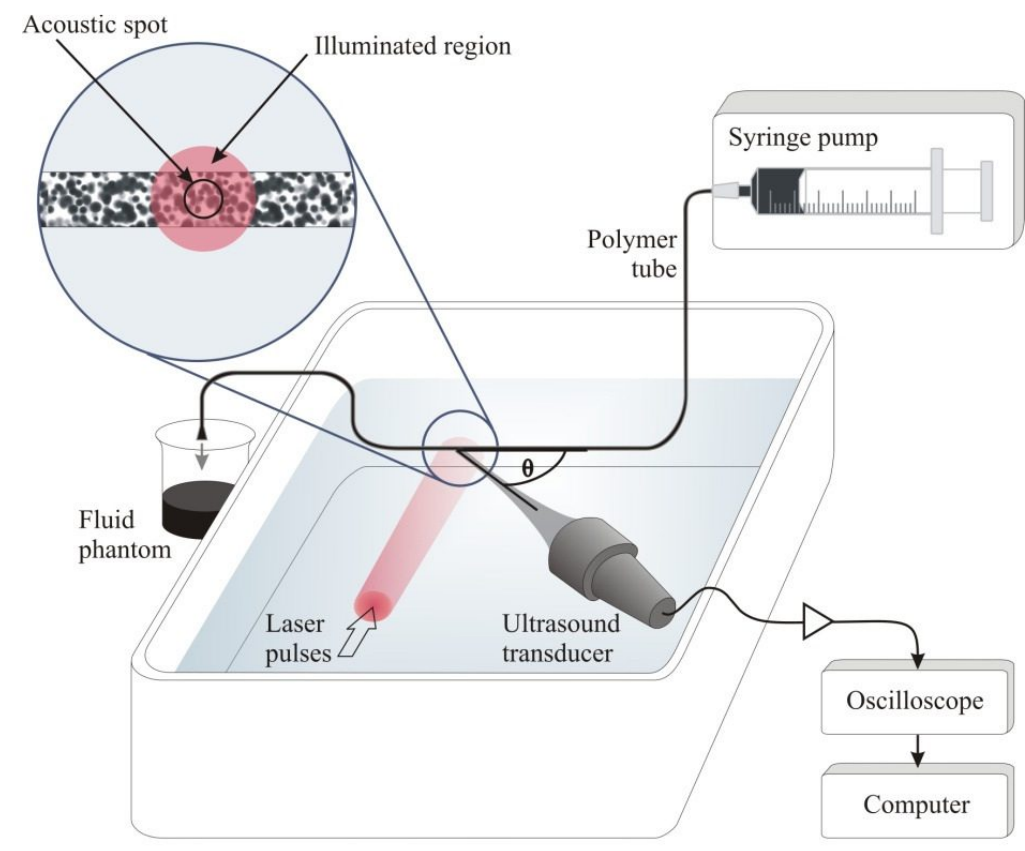

Figure 2. Experimental setup for pulsed photoacoustic Doppler flow measurement where the motion of micron-scale absorbers is used to represent blood flow. Laser pulses separated by a time $T$ are used to generate pairs of acoustic waveforms which are detected by an ultrasound receiver. The inset shows a typical distribution of the absorbers in the fluid phantoms. A large area (at least $5 \mathrm{~mm}$ diameter) of the absorbers is illuminated, but photoacoustic signals are only collected from the smaller region defined by the transducer focal spot. The signals were amplified and then captured by an oscilloscope and downloaded to a PC.

Photoacoustic waveform pairs were generated using laser pulses with pulse separations $T$ in the range $0.5 \mathrm{~ms}$ to $4 \mathrm{~ms}$. The light illuminated a large region (at least $5 \mathrm{~mm}$ in diameter) of the fluid-filled tube in a tank of water. Light absorbing particles within the target area emitted a photoacoustic wave each time they were illuminated and these waves were detected using an ultrasound transducer also immersed in the water. Four different spherically focussed transducers were investigated, with centre frequencies of $5 \mathrm{MHz}, 15 \mathrm{MHz}, 20 \mathrm{MHz}$ and $30 \mathrm{MHz}$. The signals were amplified using a 60 $\mathrm{dB}$ amplifier (Analog Modules 322-8-B-50) and captured using an oscilloscope (DSO-Tektronix TDS784D).

Experiments with the microsphere phantoms used a Q-switched Nd:YAG laser ("FQ" laser, Elforlight) pulsing at $2 \mathrm{kHz}$. The high repetition rate enabled a range of pulse separations $T$ to be investigated from a single acquisition (see section 4). However, each laser pulse provided less than $1 \mathrm{~mJ}$ at a wavelength of $1064 \mathrm{~nm}$, which was insufficient energy to generate a detectable signal in blood since the absorption coefficient of haemoglobin at $1064 \mathrm{~nm}$ is relatively low. The blood experiments therefore employed an alternative twin laser system ("PIV" laser, Litron) that incorporated a pair of Q-switched Nd:YAG lasers aligned so that they emit through a single aperture. Each laser pulsed at $15 \mathrm{~Hz}$ and provided $12 \mathrm{~mJ}$ at wavelength of $532 \mathrm{~nm}$. The two lasers were triggered using a counter/timer programmed with a DAQ card (NI PCI-6024E) to give a pre-selected time delay $T$.

\section{SIGNAL ACQUISITION AND PROCESSING}

Series of 24 waveforms were captured in real time using the FastFrame ${ }^{\mathrm{TM}}$ Segmented Memory feature of the oscilloscope. This enabled the waveforms to be concatenated in a single record and downloaded to a PC. With the FQ laser, multiple time separations $T=(0.5 n) \mathrm{ms}$ could be investigated by evaluating the unbiased cross-correlation function for different pairs of photoacoustic waveforms along the pulse train; for example, adjacent PA waveforms are 
separated by $T=0.5 \mathrm{~ms}$, alternate waveforms are separated by $T=1.0 \mathrm{~ms}$, and so on for pulse separations increasing in intervals of $0.5 \mathrm{~ms}$. The PIV laser generated laser pulse pairs and therefore the cross-correlation function could only be evaluated for successive pairs of PA waveforms.

For both lasers, each velocity measurement used four sets of 12 waveform pairs. The mean and standard deviation of 12 cross-correlation amplitudes were computed to give a mean cross-correlation function $C(t)$ with error bars equal in length to twice the standard deviation at each point. The peak of $C$ was isolated and the mean of a suitable fit (Gaussian or Weibull) used to determine the measured time shift $t_{s}$ and hence the calculated velocity value (Eq. (2)). The overall uncertainty $\Delta t_{s}$ in the measured time shift $t_{s}$ was estimated using the standard deviation values (y-axis error bars) associated with $C_{p}$ together with the sensitivity (gradient) of the curve fitting function; the corresponding uncertainty (resolution) in the velocity was calculated using the relation in Eq. (2). This was repeated for four acquisitions (four series of 24 waveforms). The final measurement $V^{\prime}$ was the mean of the four velocity values and the resolution $\pm \Delta V^{\prime} / 2$ was calculated by error propagation of the four individual resolution values.

Measurements $V^{\prime} \pm \Delta V^{\prime} / 2$ were made for speeds in the range 0 to $110 \mathrm{~mm} / \mathrm{s}$. The syringe pump could be programmed to deliver rates up to $200 \mathrm{ml} / \mathrm{hr}$ in steps of $0.01 \mathrm{ml} / \mathrm{hr}$. The pre-selected rate and the inner diameter of the tube were used to calculate the corresponding velocity in $\mathrm{mm} / \mathrm{s}$. Uncertainties in the linear velocity were based on the tolerance in the diameter of the tubing. These "known" velocity values and uncertainties were compared with those acquired via crosscorrelation of the PA waveform pairs.

\section{RESULTS AND DISCUSSION}

Sub-section 5.1 presents velocity measurements made using the different phantoms and describes the variations in the maximum measurable velocities. Factors affecting the maximum measurable velocity are discussed in sub-section 5.2: the dependence on the transducer frequency response is described in sub-section 5.2.1 and the influence of flow patterns is discussed in sub-section 5.2.2.

\subsection{Maximum measurable velocity}

Reductions in both accuracy and resolution can be attributed to loss of correlation due to the movement of a particular group of absorbers entirely out of the focal spot by the time the second laser pulse is emitted. The limiting speed $\left|V_{\text {max }}\right|$ (metres per second) beyond which correlation is lost completely can be calculated from the acoustic spot diameter ( $w$ in millimetres) and the pulse separation ( $T$ in milliseconds):

$$
\left|V_{\max }\right|=\frac{w_{p}}{T}=\frac{w}{T \sin \theta}
$$

The geometrical relationship between $w$ and $w_{p}$ is illustrated in Figure 3 .

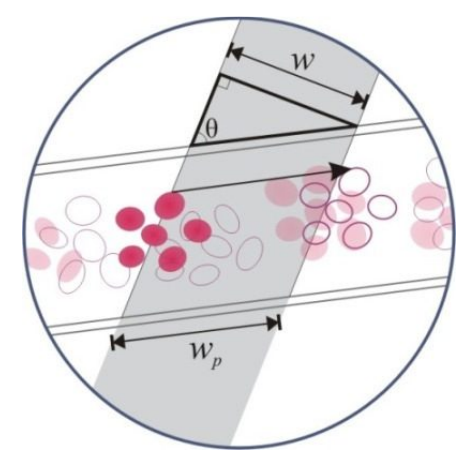

Figure 3. Demonstration of de-correlation due to the finite transducer beam width. A cluster of RBCs (represented by solid ellipses) may appear within the transducer focal beam (grey shading) at the time of the first laser pulse; if the cluster moves out of the focal beam by the time of the second laser pulse, the velocity is greater than $\left|V_{\text {max }}\right|$ and there is no correlation. 
Figure 4 illustrates how $\left|V_{\max }\right|$ and also the theoretical measurement resolution $\pm \delta V / 2$ (based on the oscilloscope sampling interval $\delta t_{s}$ ) scale inversely with pulse separation $T$. For example, velocities up to $\left|V_{\max }\right|=1 \mathrm{~m} / \mathrm{s}$ could be accurately measured with a resolution of $\pm 1 \mathrm{~mm} / \mathrm{s}$ using a laser pulse separation of $0.5 \mathrm{~ms}$; increasing the pulse separation to $5 \mathrm{~ms}$ would, theoretically, allow velocities up to a maximum of $100 \mathrm{~mm} / \mathrm{s}$ to be measured with a resolution of $\pm 0.1 \mathrm{~mm} / \mathrm{s}$.

In practice, de-correlation may occur over a distance $d_{D}$ that is shorter than $w_{p}$ to give an observed maximum measurable velocity $\left|V_{\text {max }}\right|_{\text {obs }}$ :

$$
\left|V_{\text {max }}\right|_{\text {obs }}=\frac{d_{D}}{T}
$$

Figure 5 shows velocity measurements made for four different phantoms. For the wheel phantom [Figure 5(a)], the observed maximum measurable velocity $\left|V_{\max }\right|_{\text {obs }}$ corresponds with the calculated value $\left|V_{\max }\right|$ as expected. However, for the fluid phantoms [Figure $5(\mathrm{~b}-\mathrm{d})],\left|V_{\max }\right|_{\text {obs }}$ is less than $17.5 \%$ of the calculated $\left|V_{\max }\right|$. For $T=0.5 \mathrm{~ms}$, the corresponding maximum velocities are $175 \mathrm{~mm} / \mathrm{s}, 125 \mathrm{~mm} / \mathrm{s}$ and $96 \mathrm{~mm} / \mathrm{s}$ for the $22 \mu \mathrm{m}, 17 \mu \mathrm{m}$ and $6 \mu \mathrm{m}$ spheres respectively. Since typical velocities in the microvasculature are less than $50 \mathrm{~mm} / \mathrm{s}$ these discrepancies between $\mid V_{\text {max }} l_{\text {obs }}$ and $\left|V_{\max }\right|$ do not pose a significant limitation.

Figure 6 shows results for flowing blood. Here the maximum velocity is limited to $13.5 \mathrm{~mm} / \mathrm{s}$ [Figure 6(a)] for a sample where the haematocrit was reduced to $5 \%$ of the normal physiological value $(\mathrm{Ht}=40-50 \%)$. Increasing the red cell concentration to $20 \%$ of the normal value [Figure $6(\mathrm{~b})$ ] further reduces $\left|V_{\max }\right|_{\text {obs }}$ to approximately $7 \mathrm{~mm} / \mathrm{s}$. There are also inaccuracies in the very small time shifts due to the limitations imposed by the oscilloscope sampling interval. Therefore, although reducing the pulse separation $T$ will increase the upper velocity limit, it will be at the expense of the velocity resolution. No accurate velocity measurements were achieved for haematocrits greater than $0.2 \mathrm{Ht}$.

Figure 7 summarises the differences in $\left|V_{\text {max }}\right|_{\text {obs }}$ for the different phantoms. $\left|V_{\text {max }}\right|_{\text {obs }}$ was approximately $1.6 \%, 0.9 \%$ and $0.7 \%$ of $\left|V_{\max }\right|$ for blood samples where the normal haematocrit was reduced to $0.05 \mathrm{Ht}, 0.1 \mathrm{Ht}$ and $0.2 \mathrm{Ht}$ respectively.

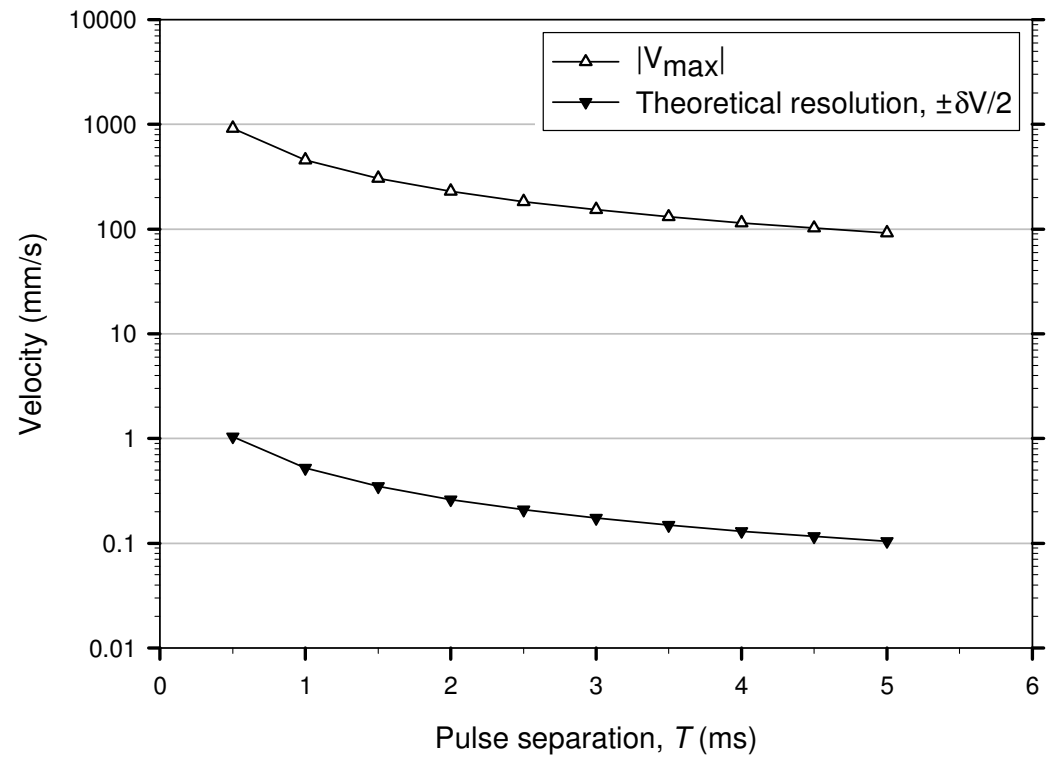

Figure 4. Scaling of theoretical resolution $\pm \delta V / 2$ and maximum measurable velocity $\left|V_{\text {max }}\right|$ with increasing pulse separation $T$. The values are calculated based on the $30 \mathrm{MHz}$ transducer $(w=0.33 \mathrm{~mm})$ with $\theta=45^{\circ}$, and a sampling interval $\delta t_{s}=0.5$ ns. 
(a)

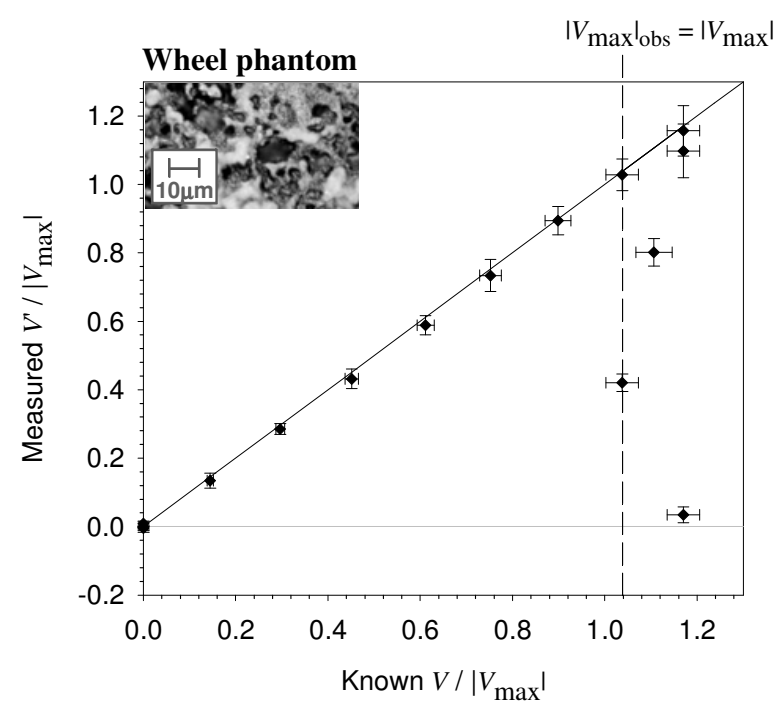

(c)

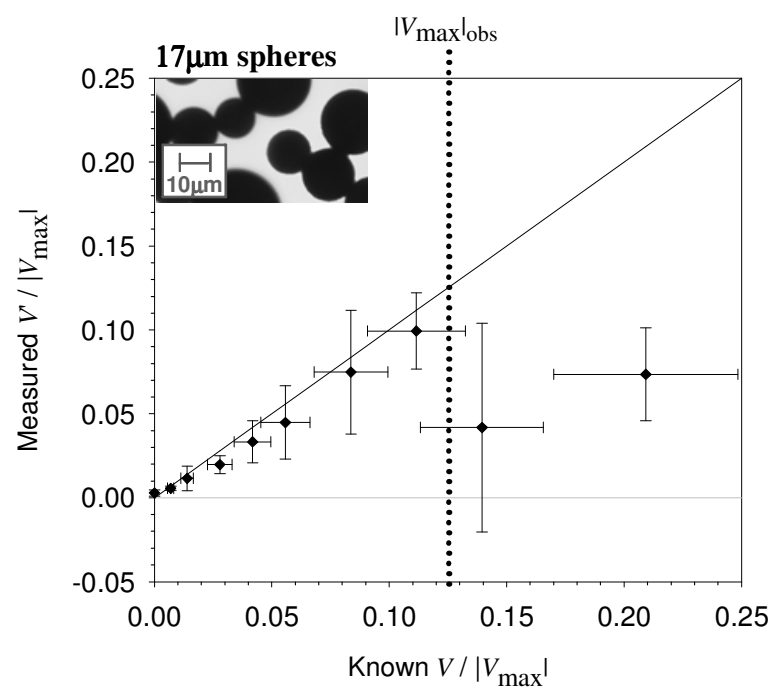

(b)

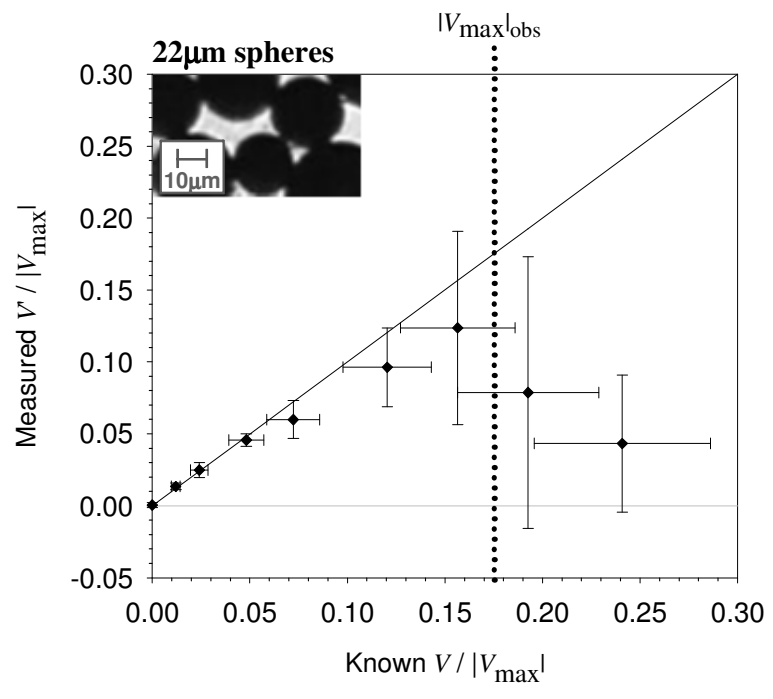

(d)

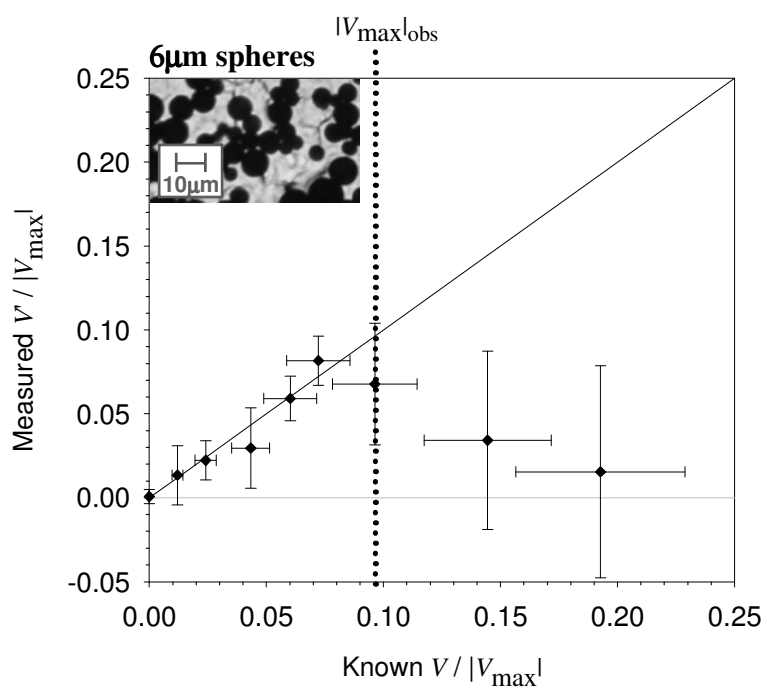

Figure 5. Velocity measurements made for the wheel phantom (a), and the three diameters of microspheres (b-d). The measurements are plotted relative to the maximum measurable velocities $\left|V_{\max }\right|$, indicated by a dashed line in (a). For the microsphere phantoms the observed maximum measurable velocities $\left|V_{\max }\right|_{\text {obs }}$, indicated by dotted lines, are less than $17.5 \%$ of the calculated $\left|V_{\max }\right|$. Calculating velocities relative to $\left|V_{\max }\right|$ normalises for the pulse separations $T$ and focal beam widths $w$. However, the range of absolute velocity values can be deduced from Figure 4. 
(a)

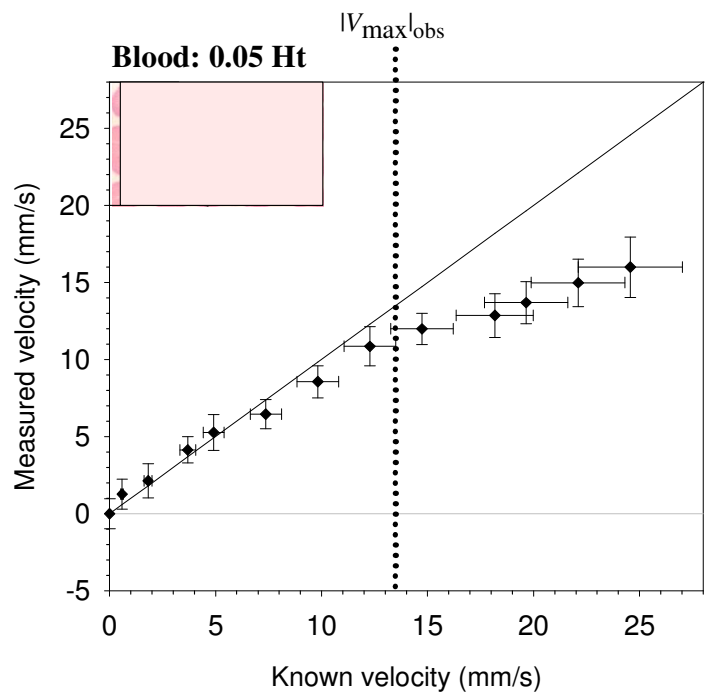

(b)

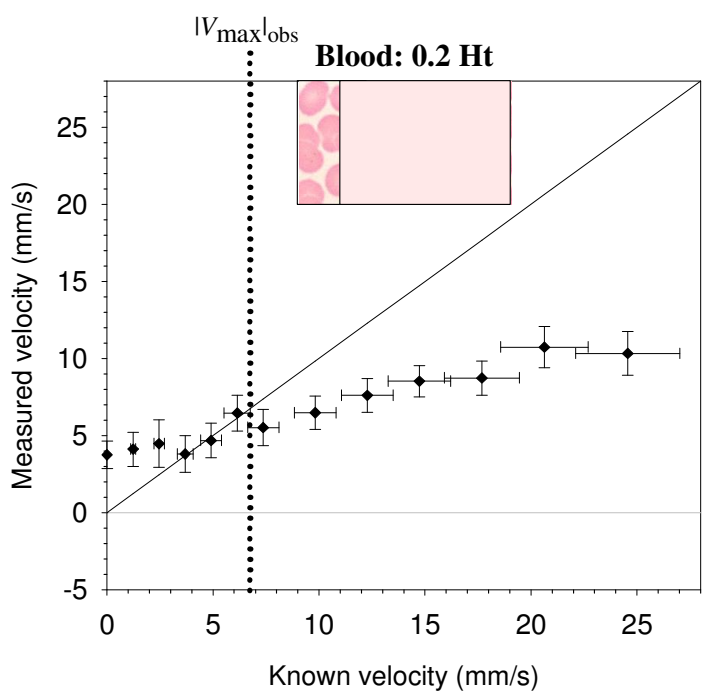

Figure 6. Velocity measurements for blood flowing in a $600 \mu \mathrm{m}$ tube. The blood was reduced to $5 \%$ of the normal haematocrit (Ht) in (a) and to $20 \%$ in (b). The data were acquired using a pulse separation $T=0.5 \mathrm{~ms}$ and a $30 \mathrm{MHz}$ transducer.

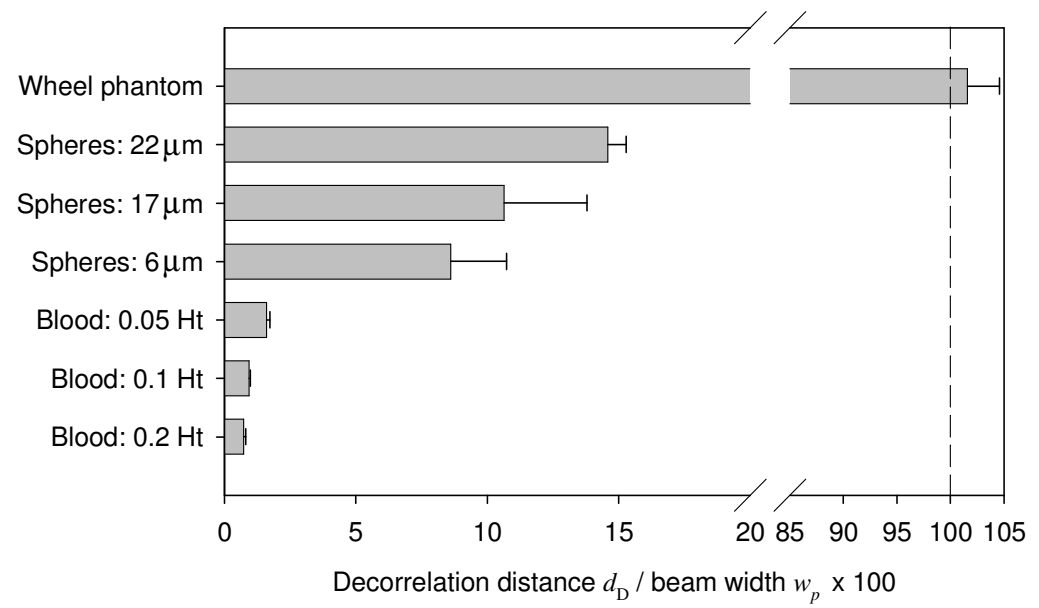

Figure 7. Minimum distances moved by the absorbers leading to de-correlation.

\subsection{Factors leading to poor correlation}

It is evident from Figure 7 that, for the fluid phantoms, there is a large discrepancy between $\left|V_{\text {max }}\right|_{\text {obs }}$ (derived from decorrelation distance $d_{D}$ ) and $\left|V_{\text {max }}\right|$ (derived from detector beam width $w$ ). This discrepancy appears to increase with reducing absorber diameter and with increasing absorber concentration. We consider here two factors that may prevent accurate measurement of velocities greater than $\left|V_{\max }\right|_{\text {obs }}$, giving rise to the discrepancy with $\left|V_{\max }\right|$. 


\subsubsection{Detector frequency response}

The frequency content of the photoacoustic signals is likely to be related to the size of the absorbers and the spacing between them [14]. Figure 8 summarises velocity measurements made for the $22 \mu \mathrm{m}$ spheres using four detectors with different centre frequencies. It is evident that detectors with a higher centre frequency enable velocity measurements to be made with better accuracy and resolution; accurate time shift measurements were not possible at all using the lowest frequency $5 \mathrm{MHz}$ detector. This supports the idea that time shifts in PA signals generated in small, highly concentrated absorbers would be better detected using ultrasound transducers with a higher centre frequency and a more broadband response.

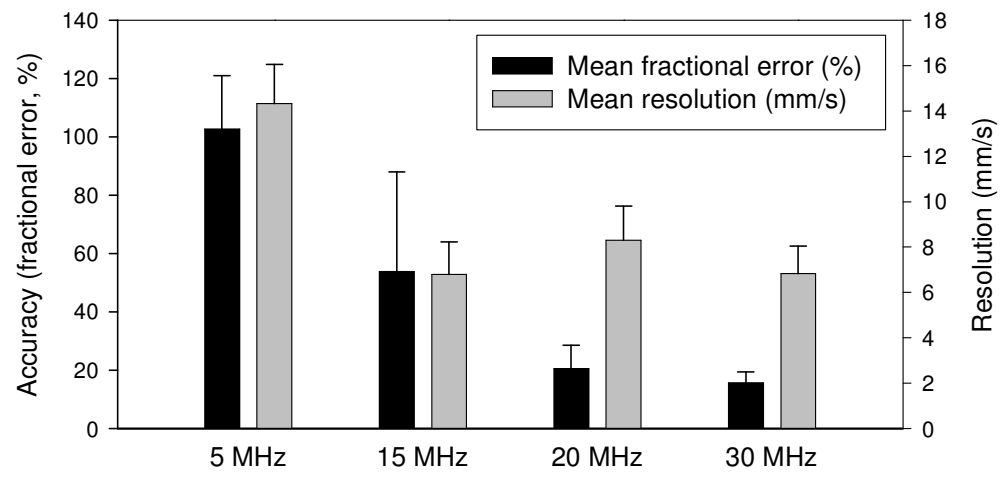

Figure 8. Comparison of the performance of different ultrasound transducers with centre frequencies of $5 \mathrm{MHz}, 15 \mathrm{MHz}, 20$ $\mathrm{MHz}$ and $30 \mathrm{MHz}$. Mean values for the accuracy (fractional error) and measurement resolution were calculated for the 22 $\mu \mathrm{m}$ spheres flowing in an $800 \mu \mathrm{m}$ tube at speeds up to $110 \mathrm{~mm} / \mathrm{s}$. The pulse separation $T$ was $0.5 \mathrm{~ms}$ and all the velocities were less than $\left|V_{\text {max }}\right|_{\text {obs }}$ and $\left|V_{\max }\right|$.

\subsubsection{Flow patterns}

The wheel phantom simulates the ideal case of plug flow; in reality the flow in the tube is more likely to be laminar, or even turbulent, where the Reynolds number Re defines the condition for turbulence. Re is given by:

$$
\operatorname{Re}=\frac{D V}{\rho \mu}
$$

where $D$ is the tube diameter, $V$ is the average velocity of flow in the tube, $\rho$ is the density of the flowing fluid and $\mu$ is the dynamic viscosity of the fluid. Re is a dimensionless number that typically takes values less than 2100 in laminar flow conditions, and values greater than 4000 for turbulent flow. Non-plug flow gives rise to axial variations in velocity and therefore poor correlation. This is evidenced by the small value of $\left|V_{\max }\right|_{\text {obs }}$ for the fluid phantoms compared to $\left|V_{\text {max }}\right|_{\text {obs }}$ for the wheel (Figure 7).

Reducing the Reynolds number may reduce turbulence giving rise to better correlation. Figure 9 compares measurements made with blood $(0.05 \mathrm{Ht})$ flowing in tubes of two different diameters; the narrower tube allows measurement of velocities up to approximately $13.5 \mathrm{~mm} / \mathrm{s}$ whereas for the wider tube there were no accurate measurements made above 3 $\mathrm{mm} / \mathrm{s}$. This suggests that the velocity range may be further extended using tubes with diameters less than $600 \mu \mathrm{m}$. 
(a)

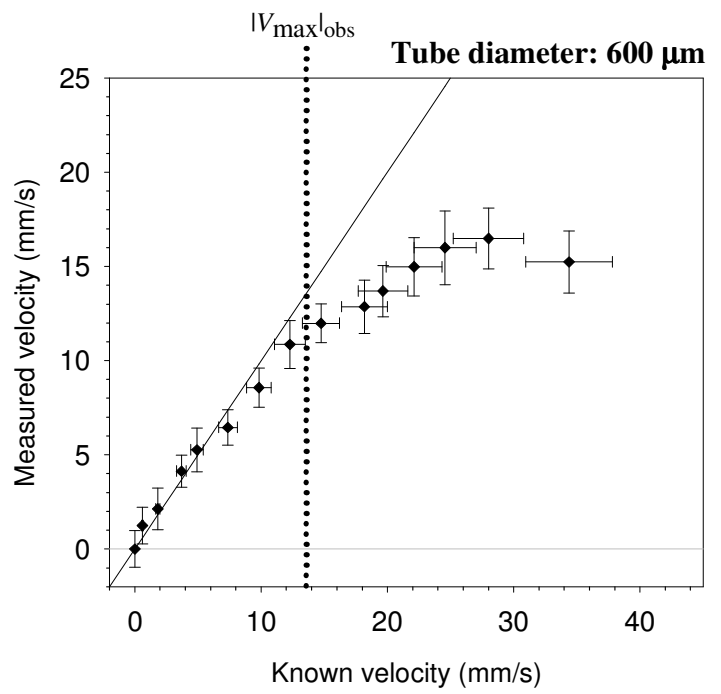

(b)

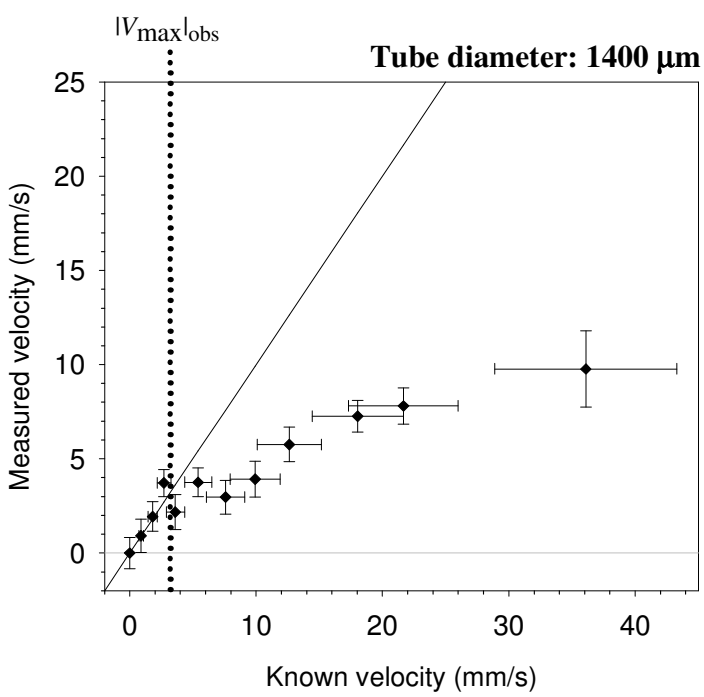

Figure 9. Velocity measurements made for blood reduced to $5 \%$ of the normal haematocrit and flowing in tubes of diameter $600 \mu \mathrm{m}$ (a) and $1400 \mu \mathrm{m}$ (b). The pulse separation $T$ was $0.5 \mathrm{~ms}$ and signals were acquired using the $30 \mathrm{MHz}$ transducer.

The nature of flow within the tubes is likely to be further complicated by the presence of the absorbers (microspheres or red blood cells). The Reynolds criteria are for a single phase fluid, but both the microsphere phantoms and the blood dilutions are effectively two-phase solid-liquid suspensions. There are cases where the size and concentration of particles in a fluid influence the critical Reynolds number at which turbulence develops [15].

To investigate the nature of flow in the tubes, they were observed under a confocal microscope: for whole blood, the velocity of blood cells at the tube boundaries were much reduced compared to the faster velocity in the tube centre. However, this boundary flow layer was not observed for the microspheres or for the lower blood cell concentrations. This suggests that at higher cell concentrations there is a greater axial variation in velocity, which may be partly due to a larger number of cells sticking to the tube walls. These cells would mask the flow through the centre of the tube, making it more difficult to make a velocity measurement representative of the faster moving cells.

\section{CONCLUSIONS}

It has been shown that a photoacoustic time shift due to moving particles can be measured via cross-correlation of pairs of photoacoustic waveforms generated using pulsed lasers and detected using an ultrasound transducer. This pulsed photoacoustic Doppler technique has proved effective for making velocity measurements in fluid phantoms consisting of suspensions of microspheres with mean diameters of $22 \mu \mathrm{m}, 17 \mu \mathrm{m}$ and $6 \mu \mathrm{m}$. Accuracies were at best within $1 \%$ of the known velocities, with resolutions of $10 \%$, for velocities up to $110 \mathrm{~mm} / \mathrm{s}$. The velocity range and resolution are scalable with excitation pulse separation $T$, but the observed maximum measurable velocity is less than $17.5 \%$ of the calculated value. For flowing blood with the concentration reduced to $0.05 \mathrm{Ht}$, the maximum measurable velocity is less than 13.5 $\mathrm{mm} / \mathrm{s}\left(1.6 \%\left|V_{\max }\right|\right)$ using a pulse separation of $0.5 \mathrm{~ms}$. A shorter laser pulse separation would extend the range of measurable velocities, but the minimum measurable velocity (resolution) would be limited by the oscilloscope time sampling interval.

The unexpectedly small values for maximum velocity $\left|V_{\text {max }}\right|_{\text {obs }}$ are due to the nature of fluid flow. When the concentration of absorbers is high the shifts in the PA signals as the absorbers move through the tube are subtle. If these subtle shifts are not detected, the correlation technique does not give accurate velocity measurements. However, we have shown (Figure 8) that the accuracy and resolution of velocity measurements improves as the detector frequency is 
increased; we therefore envisage that it will be easier to measure the subtle blood cell shifts using a higher frequency and/or more broadband detector. It is also likely that poor correlation arises from non-linear motion of the absorbers. Laminar and turbulent flow produce axial variations in velocity, whereas in the plug flow regime the velocity is uniform across the tube. Turbulence may be reduced by using narrower tubes. Alternative tube materials may also prevent adherence of the blood cells to the tube walls, which appears to be a greater problem for higher cell concentrations. We anticipate that optimisation of the detector frequency characteristics and tube phantom parameters will allow precise, spatially resolved quantification of low flow speeds such as that of blood in the microcirculation.

\section{ACKNOWLEDGEMENTS}

The authors would like to thank Elforlight and Litron for the use of their lasers.

\section{REFERENCES}

[1] Beard, P., "Biomedical photoacoustic imaging," Interface Focus 1(4), 602-631 (2011).

[2] Laufer, J., Johnson, P., Zhang, E., Treeby, B., Cox, B., Pedley, B., and Beard, P., "In vivo preclinical photoacoustic imaging of tumor vasculature development and therapy," J. Biomed. Opt. 17(5), 056016 (2012).

[3] Zhang, E. Z., Povazay, B., Laufer, J., Alex, A., Hofer, B., Pedley, B., Glittenberg, C., Treeby, B., Cox, B., Beard, P., and Drexler, W., "Multimodal photoacoustic and optical coherence tomography scanner using an all optical detection scheme for 3D morphological skin imaging," Biomed. Opt. Express 2(8), 2202-2215 (2011).

[4] Zhang, H. F., Maslov, K., Stoica, G., and Wang, L. V., "Functional photoacoustic microscopy for highresolution and noninvasive in vivo imaging," Nat. Biotechnol. 24(7), 848-851 (2006).

[5] Laufer, J., Delpy, D., Elwell, C., and Beard, P., "Quantitative spatially resolved measurement of tissue chromophore concentrations using photoacoustic spectroscopy: application to the measurement of blood oxygenation and haemoglobin concentration," Phys. Med. Biol. 52(1), 141-168 (2007).

[6] Beard, P. C., "Flow velocity measurements," UK Patent Application, WO 03/039364, (2001).

[7] Fang, H., Maslov, K., and Wang, L. V., "Photoacoustic doppler effect from flowing small light-absorbing particles," Phys. Rev. Lett. 99(18), 184501-184501-4 (2007).

[8] Sheinfeld, A., Gilead, S., and Eyal, A., "Simultaneous spatial and spectral mapping of flow using photoacoustic Doppler measurement,” J. Biomed. Opt. 15(6), 066010-066010-8 (2010).

[9] Yao, J., Maslov, K., Shi, Y., Taber, L., and Wang, L., "In vivo photoacoustic imaging of transverse blood flow by using Doppler broadening of bandwidth," Opt. Lett. 35(9), 1419-1421 (2010).

[10] Chen, S.-L., Xie, Z., Carson, P. L., Wang, X., and Guo, L. J., "In vivo flow speed measurement of capillaries by photoacoustic correlation spectroscopy," Opt. Lett. 36(20), 4017-4019 (2011).

[11] Brunker, J. and Beard, P., "Pulsed photoacoustic Doppler flowmetry using time-domain cross-correlation: accuracy, resolution and scalability," Journ. Acoust. Soc. America 3(132), 1780-1791 (2012).

[12] Brunker, J. and Beard, P., "Pulsed photoacoustic Doppler flowmetry using a cross correlation method," Proc. SPIE 7564, 756426-756426-8 (2010).

[13] Brunker, J. and Beard, P., "Pulsed photoacoustic Doppler flow measurements in blood-mimicking phantoms," Proc. SPIE 7899, 78991K-78991K-10 (2011).

[14] Hysi, E., Saha, R. K., and Kolios, M. C., "On the use of photoacoustics to detect red blood cell aggregation," Biomed. Opt. Express 3(9), 2326-2338 (2012).

[15] Matas, J.-P., Morris, J. F., Guazzelli, E., "Influence of particles on the transition to turbulence in pipe flow," Phil. Trans. R. Soc. A., 361(1806), 911-919 (2003). 